Pacific Journal of Mathematic 


\title{
ON QUASI-ISOMORPHIC INVARIANTS OF PRIMARY GROUPS
}

\author{
PAUL HILL
}

\begin{abstract}
Two primary groups $G$ and $H$ are quasi-isomorphic if there exist subgroups $G^{*}$ and $H^{*}$ of $G$ and $H$ such that $G^{*}$ and $H^{*}$ are isomorphic and such that $G / G^{*}$ and $H / H^{*}$ are bounded. The paper is concerned with properties, of primary groups, that are invariant under the relation of quasi-isomorphism. In the final section, a condition is given which is necessary and sufficient in order that the primary groups $G$ and $H$ be quasi-isomorphic in case $G$ and $H$ are both direct sums of closed groups.

The main result of the paper is that quasi-isomorphism commutes with direct decomposition for the class of primary groups whose first Ulm factors are direct sums of countable groups.
\end{abstract}

The connection between the relation of quasi-isomorphism of primary groups and their Ulm invariants was investigated by Beaumont and Pierce in [1] and [2] and by the author in [4]. Cutler [3] has recently studied properties of primary groups which are invariant under the relation of quasi-isomorphism. For example, it was proved in [3] that the property of being a direct sum of cyclic groups is invariant under quasi-isomorphism. Irwin and Richman proved, for primary groups, in [9] that the property of being a direct sum of countable groups is also a quasi-isomorphic invariant. We shall establish a decomposition theorem which contains these results as special cases; specifically, the following theorem is proved. Suppose that $G$ and $H$ are primary groups and suppose that $G=\sum G_{\lambda}$ where $G_{\lambda} / p^{\omega} G_{\lambda}$ is a direct sum of cyclic groups. If $H$ is quasi-isomorphic to $G$, then $H=\sum H_{\lambda}$ where $H_{\lambda}$ is quasi-isomorphic to $G_{\lambda}$. There is also a decomposition theorem proved for group pairs $(G, H)$ where $G$ is a direct sum of cyclic groups or a closed group and $H$ is a cobounded subgroup of $G$. In answer to a question in [3], we show that the property of being a direct sum of closed groups is not a quasi-isomorphic invariant. Even in one of the simplest cases, where $G=\bar{B}+B$, a group $H$ quasi-isomorphic to a direct sum $G$ of closed groups need not be a direct sum of closed groups. However, we show that if $G$ and $H$ are direct sums of closed groups, then $G$ and $H$ are quasi-isomorphic if they satisfy a condition which is obviously necessary; if for some bounded subgroup $B$ there exists an isomorphism from $(G+B)[p]$ onto $(H+B)[p]$ that does not alter heights more than a fixed positive 
integer $k$, then $G$ and $H$ are quasi-isomorphic.

Two abelian groups $G$ and $H$ are said to be quasi-isomorphic if there exist isomorphic subgroups $H^{*}$ and $G^{*}$ of $H$ and $G$, respectively, such that $G / G^{*}$ and $H / H^{*}$ are bounded. The notation $G \cong H$ is used to mean that $G$ and $H$ are quasi-isomorphic. We shall call a subgroup $A$ of $G$ cobounded if $G / A$ is bounded.

2. Cobounded subgroups inherit basic subgroups. Suppose that $G$ is a primary group and that $H$ is a subgroup of $G$ such that $H \supseteqq p^{n} G$ for some positive integer $n$. Let $B$ be a basic subgroup of $G$. The question was raised in [3] as to whether there exists a basic subgroup $B^{\prime}$ of $H$ such that $P^{n} B \subseteq B^{\prime} \subseteq B$, and some partial results were obtained concerning the problem. Megibben has pointed out to the author that not only does such a $B^{\prime}$ always exists but that it is unique. In fact, the following theorem can readily be established. And we shall make use of it later on in the paper.

Theorem 2.1. Suppose that $H$ is a cobounded subgroup of the primary group $G$. If $B$ is pure and dense in $G$, relative to the $p$ adic topology, then $B \cap H$ is pure and dense in $H$.

Proof. There is, of course, no proper cobounded subgroup of a divisible group. Thus $\{B, H\} / B=G / B$ and $\{B, H\}=G$, so $H / B \cap H \cong$ $\{B, H\} / B=G / B$ and $B \cap H$ is dense in $H$. Since $H$ is cobounded and since $B[p]$ is dense in $G[p]$, it is immediate that $(B \cap H)[p]$ is dense in $H[p]$. In order to prove that $B \cap H$ is pure in $H$, it suffices, according to [6], to prove that $B \cap H$ is a neat subgroup of $H$. Suppose that $p h=b$ where $h \in H$ and $b \in B \cap H$. Since $B$ is pure, there is an element $b^{\prime} \in B$ such that $p b^{\prime}=b=p h$. Now $\left(h-b^{\prime}\right)-b^{\prime \prime} \in H$ for some $b^{\prime \prime} \in B[p]$ since $B[p]$ is dense in $G[p]$ and since $H$ is cobounded. Observe that $b^{\prime}+b^{\prime \prime} \in B \cap H$ and that $p\left(b^{\prime}+b^{\prime \prime}\right)=b$. This completes the proof of the theorem.

Recall that the final rank of a basic subgroup is called the critical number of a primary group. Since a cobounded subgroup has the same final rank as the group, an immediate consequence of Theorem 2.1 is the following corollary.

CoRollary 2.2. The critical number of a primary group is a quasi-isomorphic invariant.

Theorem 2.1 also yields a refinement of Theorem 5.1 in [3].

COROLLARY 2.3. If $H$ is a cobounded subgroup of the primary group $G$ and $N$ is high in $G$, then $M=H \cap N$ is high in $H$. 
Proof. Since $N$ is high in $\mathrm{G}$, we have the relation $G[p]=$ $N[p]+G^{1}[p]$ where $G^{1}=\bigcap_{n<\omega} p^{n} G$. Thus $H[p]=M[p]+H^{1}[p]$ since $H^{1}=G^{1}$. Now the purity of $M$ in $H$ implies that $M$ is a high subgroup of $H$.

The question now arises as to whether for each pure and dense subgroup $B$ of a cobounded subgroup $H$ of the primary group $G$ there exists a pure and dense subgroup $A$ of $G$ such that $B=A \cap H$.

Theorem 2.4. If $H$ is a cobounded subgroup of the primary group $G$ and if $B$ is pure and dense in $H$, then there exists a pure and dense subgroup $A$ of $G$ such that $B=A \cap H$.

Proof. Choose $A$ maximal in $G$ with respect to $A \cap H=B$. Then $A$ is neat in $G$. We show that the socle of $A$ is dense in $G[p]$. Let $x \in G[p]$. There is an element $a \in A$ such that $x=a+h$ where $h \in H$. Since $p h \in A \cap H=B$ and since $B$ is pure, there exists $b \in B$ such that $p h=p b$. Thus

$$
x=(a+b)+(h-b) \in\{A[p], H[p]\} \subseteq\{A[p], \overline{B\lfloor p]}\} \subseteq \overline{A\lfloor p]} .
$$

By Theorem 1 in [6], $A$ is pure and dense in $G$.

Observe that $B=A \cap H$ is cobounded in $A$ since $H$ is cobounded in $G$. It follows that $B$ is a direct sum of cyclic groups if and only if $A$ is a direct sum of cyclic groups. Hence we have the following corollary.

CoROLlaRY 2.5. Let $H$ be a cobounded subgroup of the primary group $G$. The correspondence $B \rightarrow B \cap H$ is a function from the basic subgroups of $G$ onto the basic subgroups of $H$.

\section{The decompositions theorems.}

THEOREM 3.1. Suppose that the primary group $G$ is a direct sum of cyclic groups. If $H$ is a cobounded subgroup of $G$, then there exist a nonnegative integer $k$ and a decomposition $G=\sum_{i=0}^{k} G_{i}$ such that $H \cong \sum_{i=0}^{k} p^{i} G_{i}$.

Proof. Let $G[p]=P+H[p]$. Since $P$ is a discrete subsocle [7] of $G$, it supports a pure subgroup $A$ of $G$; indeed, $P$ supports a $p^{k}$-bounded direct summand $A$ of $G$ if $p^{k}(G / H)=0$. Let $G=A+G^{\prime}$ and let $H^{\prime}$ be the image of $H$ under the natural projection of $G$ onto $G^{\prime}$. Since $H \cap A=0, H^{\prime}$ is isomorphic to $H$. Furthermore, $H^{\prime}[p]=$ $G^{\prime}[p]$; hence it suffices to prove the theorem for the case $\left.H[p]=G_{i} p\right]$. Let $S=H[p]=G[p]$. Since $H$ is a $p^{k}$-cobounded subgroup of $G$, the Ulm invariants of $H$ and $G$ are related by the inequalities:

$$
\sum_{n}^{n+r} f_{H}(j) \leqq \sum_{n}^{n+r+k} f_{G}(j) \text { and } \sum_{n+k}^{n+r+k} f_{G}(j) \leqq \sum_{k}^{n+r+k} f_{H}(j)
$$


for all $n, r \geqq 0$, where $f$ is the Ulm function. It follows from an obvious modification of Lemma 1 in [4] that there exist an automorphism $\pi$ of $S$ and decompositions $S=\sum P_{n}=\sum Q_{n}$ such that the nonzero elements of $P_{n}$ and $Q_{n}$ have height $n$ in $H$ and $G$, respectively, and such that for each $x_{n} \in P_{n}$ the relation $\pi\left(x_{n}\right) \in Q_{n+i}$ holds for some nonnegative $i \leqq k$. Hence there exist decompositions $G=\sum_{i=0}^{k} G_{i}$ and $H=\sum_{i=0}^{k} H_{i}$ of $G$ and $H$ such that $H_{i} \cong p^{i} G_{i}$ for $0 \leqq i \leqq k$. Thus $H \cong \sum_{i=0}^{k} p^{i} G_{i}$.

REMARK. The isomorphism between $\mathrm{H}$ and $\sum p^{i} G_{i}$ in Theorem 3.1 cannot be replaced by set theoretic equality. This can be demonstrated by very simple examples.

CoRollary 3.2. If $H$ is a cobounded subgroup of the closed group $G$, then there exist a nonnegative integer $k$ and a decomposition $G=\sum_{i=0}^{k} G_{i}$ such that $H \cong \sum_{i=0}^{k} P^{i} G_{i}$.

Proof. Let $B$ be a basic subgroup of $G$. It follows from Theorem 2.1 that $\mathrm{B} \cap H$ is a basic subgroup of the cobounded subgroup $H$ of $G$. Since $B \cap H$ is a cobounded subgroup of $B$, Theorem 3.1 implies that there exist a nonnegative integer $k$ and a decomposition $B=\sum_{i=0}^{k} B_{i}$ such that $B \cap H \cong \sum_{i=0}^{k} p^{i} B_{i}$. Thus $G=\bar{B}=\sum_{i=0}^{k} \bar{B}_{i}$ and $H \cong \sum_{i=0}^{k} p^{i} \bar{B}_{i}$ since $G$ and $H$ are closed.

Our main decomposition theorem concerning quasi-isomorphism is the following.

THEOREM 3.3. Suppose for the primary group $G$ that $G=\sum_{\lambda \in_{\Lambda}} G_{\lambda}$ where $G_{\lambda} / p^{\omega} G_{\lambda}$ is a direct sum of countable groups for each $\lambda \in \Lambda$. If $H$ is quasi-isomorphic to $G$, then $H=\sum_{\lambda \in_{A}} H_{\lambda}$ where $H_{\lambda}$ is quasiisomorphic to $G_{\lambda}$ for each $\lambda \in \Lambda$.

Proof. Since decompositions lift, for arbitrary G, from $p^{n} G$ to $G$, it suffices to prove the theorem for the case that $H$ is a cobounded subgroup of $G$. Suppose that $p^{k} G \subseteq H \subseteq G$. In case $G$ is a direct sum of cyclic groups, the theorem follows from Theorem 3.1 and the isomorphic refinement theorem for direct sums of cyclic groups. In fact, since $H$ is $p^{k}$-cobounded in $G$, we can write $H=\sum_{\lambda_{1} \epsilon_{A}} H_{\lambda}$ where $H_{\lambda}$ is isomorphic to a $p^{k}$-cobounded subgroup of $G_{\lambda}$. Thus, in the general case, we can write $H / p^{\omega} H=\sum_{\lambda \epsilon_{A} H_{\lambda}^{*}}$ where $H_{\lambda}^{*}$ is isomorphic to a $p^{k}$-cobounded subgroup of $G_{\lambda} / p^{\omega} G_{\lambda}$. For each $\lambda \in \Lambda$, let $G_{\lambda}^{*}$ be a cobounded subgroup of $G_{\lambda}$ such that $G_{\lambda}^{*} / p^{\omega} G_{\lambda} \cong H_{\lambda}^{*}$. Set $G^{*}=\sum_{\lambda} \in_{\lambda} G_{\lambda}^{*}$. Now $p^{\omega} G^{*}=p^{\omega} G=p^{\omega} H$, and $G^{*} / p^{\omega} G^{*}=G^{*} / p^{\omega} G=\sum_{\lambda \epsilon_{\Lambda}}\left(G_{\lambda}^{*} / p^{\omega} G_{\lambda}\right)=$ $\sum_{\lambda \in A} H_{\lambda}^{*}=H / p^{\omega} H$. It follows that $H \cong G^{*}$ by the uniqueness theorem [8] of Hill and Megibben, and the theorem is proved. 
DEFinition 3.4. A primary group $G$ is said to be a pillared group if $G / p^{\omega} G$ is a direct sum of cyclic groups.

Since the property of being a direct sum of cyclic groups is a quasi-isomorphic invariant, the property of being a pillared group is a quasi-isomorphic invariant. Our Theorem 3.3 shows that for pillared groups quasi-isomorphism is compatible with direct decompositions. We conclude this section with the following consequence of Theorem 3.3 .

Corollary 3.5. Suppose that $G=\sum_{\lambda \epsilon_{A} G_{\lambda}}$ is a pillared group.

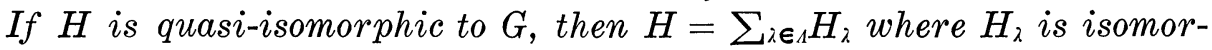
phic to a direct summand of $G_{\lambda}+C_{\lambda}$ and $C_{\lambda}$ is a direct sum of cyclic groups.

4. Some quasi-isomorphic variants. As we have mentioned, it was established in [3] and [9] that the property of being a direct sum of cyclic groups and the property of being a direct sum of countable groups are invariant under the relation of quasi-isomorphism of primary groups ; this is also an immediate consequence of Corollary 3.5. Cutler observed in [3] that the property of being a closed group is a quasiisomorphic invariant, but he left open the following question. If $G$ is a direct sum of closed groups and if $H$ is quasi-isomorphic to $G$, does $H$ have to be a direct sum of closed groups? The next theorem shows that the answer is negative.

Let $A=\sum\left\{a_{n}\right\}$ and $B=\sum\left\{b_{n}\right\}$ be copies of the standard basic subgroup, that is, let $\left\{a_{n}\right\}$ and $\left\{b_{n}\right\}$ denote cyclic groups of order $p^{n}$. Denote by $\bar{A}$ the closed group $\sum_{T}^{*}\left\{a_{n}\right\}$, the torsion completion of $A$. We want to consider the group $G=\bar{A}+B$ and a certain cobounded subgroup of $G$.

Theorem 4.1. The group $G=\bar{A}+B$, where $A$ and $B$ are copies of the standard basic subgroup, has a cobounded subgroup $H$ with the following properties.

(1) $H$ is not pure-complete.

(2) $H$ is not semi-complete.

(3) $H$ is not a direct sum of closed groups.

Proof. Define $H=\left\{p G, a_{1}, a_{n+1}+b_{n}\right\}_{n<\omega}$. Let $S=\bar{A}[p]$, and observe that $S \subseteq H$. We show that $S$ does not support a pure subgroup of $H$. Assume that $S$ does support a pure subgroup $K$ of $H$. Since each element of $S$ has the same height in $H$ as in $G$, it follows that $K$ is pure in $G$. Since $K[p]=\bar{A}[p], K$ is a closed group; hence $K$ is a direct summand of $G$. In fact, we have the decompositions $G=$ $K+B$ and $H=K+(H \cap B)$. It is easily verified that $H \cap B=p B$, 
so we have the equation $H=K+p B$. The defining equation for $H$ and the above decompositions imply that $\left\{p K+p B, a_{1}, a_{n+1}+b_{n}\right\}_{n<\omega}=$ $K+p B$. Thus, for each positive integer $n$,

$$
p^{n}\left(a_{n+2}+b_{n+1}\right)=p^{n} k_{(n)}+p^{n+1} b_{(n)}
$$

where $k_{(n)} \in K$ and $b_{(n)} \in B$.

Define $s(1)=1$ and suppose that a positive integer $s(i)$ has been chosen for $i \leqq n$ such that $s(1)<s(2)<\cdots<s(n)$. Choose $s(n+1)>s(n)$ such that $b(n) \in \sum_{i<s(n+1)}\left\{b_{i}\right\}$. Since $p^{2}\left(p^{n} k_{(n)}\right)=0$ for each $n$ and since $K$ is closed, $\sum p^{s(n)} k_{(s(n))}$ must converge in $K$. Since

$$
p^{n} k_{(n)}=p^{n} a_{n+2}+\left(p^{n} b_{n+1}-p^{n+1} b_{(x)}\right)
$$

and since $G=\bar{A}+B$, it follows that $\sum\left(p^{s(n)} b_{s(n)+1}-p^{s(n)+1} b_{(s(n)}\right)$ must converge in $B$. However, this is impossible since, for each positive integer $n$, the projection of the limit onto $\left\{b_{s(n)+1}\right\}$ is $p^{s(n)} b_{s(n)+1} \neq 0$. Thus $\mathrm{S}$ does not support a pure subgroup of $H$, and we have verified (1).

Assume that $H$ is a direct sum of closed groups. Then $H$ is a direct sum of a countable number of closed groups since $G$, and therefore $H$ by Theorem 2.1, has a countable basic subgroup. It follows from Theorem 5.6 in [7] that a direct sum of a countable number of closed groups is pure-complete. Since we have already verified (1), we conclude that $H$ is not a direct sum of closed groups. Furthermore, every semi-complete group [10] is a direct sum of closed groups, so the theorem is proved.

COROLLARY 4.2. The property of being pure-complete is not a quasi-isomorphic invariant.

COROLLARY 4.3. The property of being semi-complete is not a quasi-isomorphic invariant.

COROLLARY 4.4. The property of being a direct sum of closed groups is not a quasi-isomorphic invariant.

In view of Corollary 4.4, a natural question is: what are the groups that are quasi-isomorphic to direct sums of closed groups? In this connection, we make the following observation.

Proposition 4.5. If the primary group $G$ is quasi-isomorphic to a direct sum of closed groups, then $G[p]=\sum S_{2}$ where (1) $S_{2}$ is com. plete and (2) there exists a fixed positive integer $k$ such that height $\left(x_{1}+x_{2}+\cdots+x_{n}\right) \leqq$ height $\left(x_{i}\right)+k$ if $x_{i} \in S_{\lambda_{i}}$ for distinct $\lambda_{1}, \lambda_{2}, \cdots, \lambda_{n}$. 
Proof. Suppose that $G$ is quasi-isomorphic to a direct sum of closed groups. Then there are closed groups $H_{\lambda}$ such that $G \doteq \sum_{\lambda \epsilon_{1}} H_{\lambda}$ and such that there exists an isomorphism $\pi$ from $G[p]$ onto $\sum_{\lambda \in A} H_{\lambda}[p]$ that does not alter heights (computed in $G$ and $H=\sum H_{\lambda}$ ) more than a fixed positive integer $k$. Defining $S_{\lambda}$ by the equation $\pi\left(S_{\lambda}\right)=H_{\lambda}[p]$, we have $S_{\lambda}$ 's which satisfy the conditions (1) and (2).

5. Quasi-isomorphism of direct sums of closed groups. Although two primary groups $G$ and $H$ can be quasi-isomorphic with one a direct sum of closed groups and the other not, there is a particularly simple criterion which determines whether $G$ and $H$ are quasi-isomorphic in case both $G$ and $H$ are direct sums of closed groups.

THEOREM 5.1. Suppose that the primary groups $G$ and $H$ are direct sums of closed groups. Then $G$ and $H$ are quasi-isomorphic if and only if there is a bounded group $B$ and an isomorphism between $(G+B)[p]$ and $(H+B)[p]$ that does not alter heights more than a fixed positive integer $k$.

Proof. If $G \cong H$, there exist $p^{k}$-cobounded subgroups $G^{*}$ and $H^{*}$ of $G$ and $H$, respectively, such that $G^{*} \cong H^{*}$. Define $\pi$ from $G^{*}[p]$ onto $H^{*}[p]$ as the restriction of some isomorphism $\varphi$ from $G^{*}$ onto $H^{*}$. Let $G[p]=P+G^{*}[p]$ and $H[p]=Q+H^{*}[p]$. The height of a nonzero element in $P$ or $Q$ does not exceed $k$. For a sufficiently large $p$-bounded group $B,|P+B|=|Q+B|$ and $\pi$ can be extended to an isomorphism from $(G+B)[p]$ onto $(H+B)[p]$ having the desired property that heights are not altered more than $k$.

For the proof of the nontrivial half of the theorem, we may assume that $B=0$ since the relation of quasi-isomorphism is transitive. Thus suppose that $G=\sum_{\lambda \epsilon_{A}} G_{\lambda}$ and $H=\sum_{\mu \epsilon_{M}} H_{\mu}$ are direct sums of closed groups and that $\pi$ is an isomorphism from $G[p]$ onto $H[p]$ that does not alter heights more than $k$. We wish to show that $G \cong H$. Since $G_{\lambda}$ or $H_{\mu}$ can be zero, there is no loss of generality in assuming that $\Lambda=M$. Thus we shall make this assumption. If $\Lambda$ is countable, then $G+H$ is pure-complete and the proof that $G \cong H$ is essentially the same as the proof of Corollary 1 in [4]. Let $K$ be a pure subgroup of $G+H$ such that $K[p]=\{(x, \pi(x)): \quad x \in G[p]\}$. Then $K$ is a subdirect sum of isomorphic cobounded subgroups $G^{*}$ and $H^{*}$ of $G$ and $H$, respectively.

We now assume that $\Lambda$ is uncountable and proceed by induction on the cardinality of $\Lambda$. According to the next theorem, there are decompositions $G=\sum_{\lambda \epsilon_{\Lambda}} G^{*}{ }_{\lambda}$ and $H=\sum_{\lambda \in{ }_{\lambda}} H_{\lambda}^{*}$ of $G$ and $H$ into closed groups such that, for each $\lambda \in \Lambda$, there exists a countable subset $M_{\lambda}$ 
of $\Lambda$ having the property that

$$
\varphi\left(G_{\lambda}[p]\right) \subseteq \sum_{M_{\lambda}} H_{\mu} \text { and } \varphi^{-1}\left(H_{\lambda}[p] \subseteq \sum_{M_{\lambda}} G_{\mu}\right.
$$

for some isomorphism $\varphi$ between $G[p]$ and $H[p]$ that alters heights no more than $k$. It follows that there is an ascending chain

$$
\Lambda_{0} \subseteq \Lambda_{1} \subseteq \cdots \subseteq \Lambda_{\alpha} \subseteq \cdots, \Lambda_{\alpha} \subseteq \Lambda,
$$

that leads up to $\Lambda$ such that $\varphi\left(\sum_{\Lambda_{\alpha}} G_{\lambda}[P]\right)=\sum_{\Lambda_{\alpha}} H_{\lambda}[p]$ and $\left|\Lambda_{\alpha}\right|<|\Lambda|$. We conclude that there exist decompositions

$$
G=\sum_{\alpha} \sum_{r_{\alpha}} G_{i}^{*} \text { and } H=\sum_{\alpha} \sum_{r_{\alpha}} H_{i}^{*}
$$

such that there is an isomorphism from $\sum_{I_{\alpha}} G_{i}^{*}[p]$ onto $\sum_{I_{\alpha}} H_{i}^{*}$ that does not alter heights more than $k$ and such that $\left|I_{\alpha}\right|<|\Lambda|$. The proof of the theorem is finished by an application of the induction hypothesis; however, we owe a proof of the following theorem.

THEOREM 5.2. For the primary groups $G$ and $H$, suppose that $G=\sum_{\lambda \epsilon_{A}} G_{\lambda}$ and $H=\sum_{\lambda \epsilon_{\Lambda} H_{\lambda}}$ where $G_{\lambda}$ and $H_{\lambda}$ are closed groups. If $\pi$ is an isomorphism from $G[p]$ onto $H[p]$ that alters heights no more than $k$, then there are decompositions $G=\sum_{i \epsilon_{\Lambda}} G_{\lambda}^{*}$ and $H=\sum_{\lambda_{i} \in_{\Lambda}} H_{\lambda}^{*}$ of $G$ and $H$ into closed groups and an isomorphism $\varphi$ from $G[p]$ onto $H[p]$ that alters heights no more than $k$ such that, for each $\lambda \in \Lambda$, there exists a countable subset $M_{\lambda}$ of $\Lambda$ such that

$$
\varphi\left(G_{\lambda}^{*}[p]\right) \subseteq \sum_{M_{\lambda}} H_{\lambda}^{*} \text { and } \varphi^{-1}\left(H_{\lambda}^{*}[p]\right) \subseteq \sum_{M_{\lambda}} G_{\lambda} \text {. }
$$

Proof. The proof is similar to the proof of Theorem 2 in [5], except in the present case most of the details are simplier. Here, we include only an outline of the proof. The following lemma is essential.

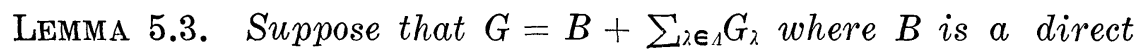
sum of cyclic groups and $G_{2}$ is closed for each $\lambda \in \Lambda$. If $H$ is a closed group and if $\pi$ is an isomorphism from $H[p]$ into $G[p]$ that does not decrease heights more than a fixed positive integer $k$, then there exists a positive integer $n$ such that $\pi\left(p^{n} H \cap H[p]\right)$ is contained in a finite number of the groups $G_{\lambda}$.

Applying Lemma 5.3 to $\pi$ and $\pi^{-1}$ and working back and forth between $G_{\lambda}$ and $H_{\lambda}$, we obtain decompositions $G=A+\sum_{\lambda_{1} \in_{\lambda}} G_{\lambda}^{\prime}$ and $H=B+\sum_{\lambda \in \epsilon_{A}} H_{\lambda}^{\prime}$ such that (i) $A$ and $B$ are direct sums of cyclic groups, (ii) $\pi\left(\sum_{\lambda \in \Lambda} G_{\lambda}^{\prime}[\mathrm{p}]\right)=\sum_{\lambda \in \epsilon_{A}} H_{\lambda}^{\prime}[\mathrm{p}]$, (iii) For each $\lambda$, there exists a finite subset $M_{\lambda}$ of $\Lambda$ such that

$$
\pi\left(G_{\lambda}^{\prime}[p]\right) \cong \sum_{M_{\lambda}} H_{\mu}^{\prime} \text { and } \pi^{-1}\left(H_{\lambda}^{\prime}[p]\right) \cong \sum_{M_{\lambda}} G_{\mu}^{\prime},
$$


(iv) there is an isomorphism $\varphi$ from $A[p]$ onto $B[p]$ that alters heights no more than $k$. This essentially finishes the proof of Theorem 5.2.

\section{REFERENCES}

1. R. A. Beaumont and R. S. Pierce, Quasi-isomorphism of p-groups, Proceedings of the Colloquium on Abelian Groups, Budapest, 1964.

2. - Quasi-isomorphism of direct sums of cyclic groups, Acta Math. Acad. Sci. Hung., 16 (1965).

3. D. O. Cutler, Quasi-isomorphism for infinite abelian p-groups, Pacific. J. Math. (1966).

4. Paul Hill, Quasi-isomorphism of primary groups Michigan Math. J. 13 (1966).

5. - The isomorphic refinement theorem for direct sums of closed groups (to appear)

6. P. Hill and C. K. Megibben, Minimal pure subgroups in primary groups, Bull. Soc. Math. France 92 (1964).

7. - On primary groups with countable basic subgroups, Trans. Amer. Math. Soc. (1966).

8. - Extending automorphisms and lifting decompositions in abelian groups (to appear).

9. J. M. Irwin and F. Richman, Direct sums of countable groups and related concepts, Journal of Algebra 2 (1965).

10. G. Kolettis, Semi-complete primary abelian groups, Proc. Amer Math. Soc. 11 (1960).

Received October 10, 1966. This research was supported by NSF Grant GP-5875. 



\section{PACIFIC JOURNAL OF MATHEMATICS}

\section{EDITORS}

\section{H. SAMELSON}

Stanford University

Stanford, California

J. P. JANS

University of Washington

Seattle, Washington 98105

\section{J. DugundJI}

University of Southern California Los Angeles, California 90007

RICHARD ARENS

University of California

Los Angeles, California 90024

\section{ASSOCIATE EDITORS}

E. F. BECKENBACH
B. H. NeUmanN

\section{SUPPORTING INSTITUTIONS}

UNIVERSITY OF BRITISH COLUMBIA CALIFORNIA INSTITUTE OF TECHNOLOGY

UNIVERSITY OF CALIFORNIA

MONTANA STATE UNIVERSITY

UNIVERSITY OF NEVADA

NEW MEXICO STATE UNIVERSITY

OREGON STATE UNIVERSITY

UNIVERSITY OF OREGON

OSAKA UNIVERSITY

UNIVERSITY OF SOUTHERN CALIFORNIA

\author{
STANFORD UNIVERSITY \\ UNIVERSITY OF TOKYO \\ UNIVERSITY OF UTAH \\ WASHINGTON STATE UNIVERSITY \\ UNIVERSITY OF WASHINGTON \\ AMERICAN MATHEMATICAL SOCIETY \\ CHEVRON RESEARCH CORPORATION \\ TRW SYSTEMS \\ NAVAL ORDNANCE TEST STATION
}

Mathematical papers intended for publication in the Pacific Journal of Mathematics should be typewritten (double spaced). The first paragraph or two must be capable of being used separately as a synopsis of the entire paper. It should not contain references to the bibliography. Manuscripts may be sent to any one of the four editors. All other communications to the editors should be addressed to the managing editor, Richard Arens at the University of California, Los Angeles, California 90024.

50 reprints per author of each article are furnished free of charge; additional copies may be obtained at cost in multiples of 50 .

The Pacific Journal of Mathematics is published monthly. Effective with Volume 16 the price per volume ( 3 numbers) is $\$ 8.00$; single issues, $\$ 3.00$. Special price for current issues to individual faculty members of supporting institutions and to individual members of the American Mathematical Society: $\$ 4.00$ per volume; single issues $\$ 1.50$. Back numbers are available.

Subscriptions, orders for back numbers, and changes of address should be sent to Pacific Journal of Mathematics, 103 Highland Boulevard, Berkeley 8, California.

Printed at Kokusai Bunken Insatsusha (International Academic Printing Co., Ltd.), 7-17, Fujimi 2-chome, Chiyoda-ku, Tokyo, Japan.

\section{PUBLISHED BY PACIFIC JOURNAL OF MATHEMATICS, A NON-PROFIT CORPORATION}

The Supporting Institutions listed above contribute to the cost of publication of this Journal, but they are not owners or publishers and have no responsibility for its content or policies. 


\section{Pacific Journal of Mathematics}

\section{Vol. 22, No. $2 \quad$ February, 1967}

Paul Frank Baum, Local isomorphism of compact connected Lie groups ....

Lowell Wayne Beineke, Frank Harary and Michael David Plummer, On the

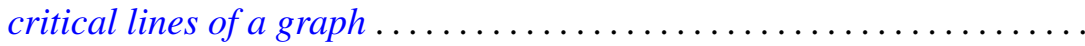

Larry Eugene Bobisud, On the behavior of the solution of the telegraphist's equation for large velocities .......................... 213

Richard Thomas Bumby, Irreducible integers in Galois extensions . . . . . . 221

Chong-Yun Chao, A nonimbedding theorem of nilpotent Lie algebras ..... 231

Peter Crawley, Abelian p-groups determined by their Ulm sequences ...... 235

Bernard Russel Gelbaum, Tensor products of group algebras ........... 241

Newton Seymour Hawley, Weierstrass points of plane domains .......... 251

Paul Daniel Hill, On quasi-isomorphic invariants of primary groups . . . . . 257

Melvyn Klein, Estimates for the transfinite diameter with applications to confomral mapping ................................ 267

Frederick M. Lister, Simplifying intersections of disks in Bing's side approximation theorem ............................. 281

Charles Wisson McArthur, On a theorem of Orlicz and Pettis ........... 297

Harry Wright McLaughlin and Frederic Thomas Metcalf, An inequality for

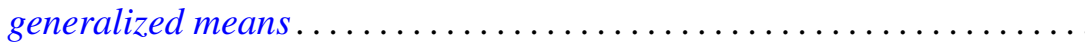

Daniel Russell McMillan, Jr., Some topological properties of piercing

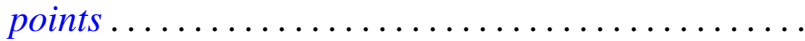

Peter Don Morris and Daniel Eliot Wulbert, Functional representation of topological algebras .

Roger Wolcott Richardson, Jr., On the rigidity of semi-direct products of Lie algebras..................................

Jack Segal and Edward Sandusky Thomas, Jr., Isomorphic

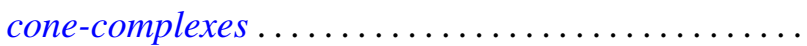

Richard R. Tucker, The $\delta^{2}$-process and related topics.... 